\title{
ORIGINALIEN
}

\section{Die Beziehungen zwischen Thrombose des Sinus cavernosus und Sinus longitudinalis superior und Erkrankungen der Nase und Nasennebenhöhlen.}

\author{
Von
}

\author{
Dr. Kiyoshi Uta. \\ Aus dem pathologischen Institüt der Universitāt Eerlin (Charité).
}

In der Hals-Nasen- und Ohrenklnik der Charité, Berlin habe ich 77 Fälle der rhinogenen Gehirnkomplikationen beobachtet. Von diesen 77 Fällen der rhinogenen Gehirnkoplikationen zeigten sich 9 Thrombose des Sinus cavernosus und 5 Thrombose des Sinus longitudinalis superior, und von 352 Fällen der otogenen Gehirnkomplipationen habe ich zugleich 7 Fälle von der isolierten Cavernosusthrombose beobachtet. Über diese otogenen isolierten Cavernosusthrombosen habe ich schon in Virchows Archiv Band 249. 1924 genau geschrieben.

Die Thrombose des Sinus cavernosus und des Sinus longitudinalis superior im Anschluss an Nasenerkrangungen kormt nicht häufig vor. Diese Sinus sind nur selten der Sitz einer primären, sondern zumeist der einer fortgeleiteten sekun. dären Thrcmbose. Zunächst will ich eine Schilderung der Gefässe der Nase und der Nebenhöhlen geben. Das reiche Venennetz der Nase spielt hierbei ja mitunter eine verhängnisvolle Rolle.

\section{Anatomie.}

\section{A. Arterin, die bei der Versorgung der Nase in Betracht kommen.}

I. Äste der A. ophthalmica.

a) Aa. ethmoidales (anterior et posterior).

Die schwächere, hintere A. ethmoidalis posterior dringt durch das Foramen ethmoidale posterius besonders zu den Siebbeinzellen, meist auch noch mit kleinen Zweigen zur Nasenscheidewand und zur harten Hirnhaut. Die stärkere vordere 
A. ethmoidalis anterior tritt durch das Foramen ethmoidale anterius in die Schädelhöhle, gibt in der vorderen Schädelgrube die A. meningea anterior ab und gelangt von hier durch ein vorderes Loch der Lamina ceribrosa zur Nasenhöhle, wo sie mit latıralen und medialen Endzweigen teils an die Seitanwand, teils an die Scheidenwand gelangt, sich hier verästelt und mit den hinteren Nasengefässen verbindungen eingeht. Nasale $Z$ weige von hier versorgen auch die mitteleren und vorderen Siebbeinzellen nebst dem Sinus frontalis.

b) A. dorsalis nasi.

Der Endast der A. ophthalmica, A. dorsalis nasi läuft über das Ligamentum palpebrale mediale nach vorn zur Nasenwurzel, von wo sie sich verzweigt und mit den benachbarten Arterien, namentlich mit der A. angularis aus der A. maxillaris externa sich verbindet.

2) Äste der A. sphenopalatina, Endast der A. maxillaris interna, treten auch durch das Foramen sphenopalatinum in den oberen hinteren Teil der Nasenhöhle und teilen sich in mehrere Äste, davon kommen in Betracht :

a) A. nasalis posterior lateralis, hält sich an der Seitenwand der Nasenhöhle, schikt sagittale $Z$ weigen zu beiden Flächen der Muscheln und zu den Nasengängen bis zum Boden der Nasenhöhle, in die Schleimhaut der Stirn-u. Kieferhöhle, sowie der Siebbeinzellen.

b) A. nasalis posterior septi betritt an der Decke der Nasenhöhle in die Nasenscheidewand und versorgt eienen grossen Teil des Septums.

3) Äste der A. maxillaris externa.

Die äussere Nase besitzt ein mächti gen das der inneren Nase nicht abgeschlossen ist. Die Arterien stammen grösstenteils aus der A. maxillaris externa. Di: oberen Äste verlaufen über den Nasenflügel und die unteren Äste nach dem Nasenflügelrande und inosculieren in die benachbarten Arterien, so auch in die A. septi nasium.

Der Endast der A. maxillaris, die A. angularis steht an der Nasenwurzel in Verbindung mit der A. dorsalis nasi, dem Endast der A. ophthalmica.

\section{B. Venen.}

I. Die Venen der äusseren Nase :

Die Venen der äusseren Nase anastomosieren mit den Venen des Naseninnern. Sie sind an der Nasenspitze zahlreich und vielfach untereinander anastomosierend. 
Aus diesem Venennetz gehen drei bis vier grössere Stämme hervor, die zwischen den beiden Venae angulares ausgesfannt sind. Die $V$. angularis steht mit den Vv. frontalis und supraorbitalis in Verbindung und zusammen mit ihnen mit dem vorderen Ende der V. ophthalmica superior. Anderseits steht das Venennetz der äusseren Nase mit den Venen der seitlichen Gesichtspartien, den Ästen der V. facialis anterior in Verbindung.

Das Blut der äusseren Nase kann mithin auf mehreren Wegen in das Schädelinnere gelangen. Einmal kann es mit der V. ophthalmica durch die Fissura orbitalis superior in den Sinus cavernosus gelangen, dann auf dem Wege über die V. facialis profunda und den Plexus perygoideus durch das Emissarium foraminis laceri und durch das Emissarium foraminis ovalis ebenfalls mit den Sinus cavernosus sich vereinigen.

II. Das Gebiet der V. ophthalmica, soweit es für die Nase in Betracht kommt.

Vorn an der medialen oberen Seite des Augapfels gelegen, wendet sie sich weiter hinten über den Sehnerven lateralwärts, gelangt in die Fissura orbitalis superior und erweitert sich hier zum Sinus ophthalmicus.

a) Am medialen Augenwinkel liegt ein Venennetz, aus welchem sich der Stamm der V. ophthalmica bildet. Dieses Venennetz steht andererseits mit den Venen des Gesichts in Verbindung und zwar mit der V. angularis, dem Anfang der V. facialis anteriores, mit der V. frontatis und der V. supraorbitalis, welche es untereinander in Verbindung setzt.

b) Die Vv. ethmoidales (anterior et posterior) sind Seitenäste der Vv. ophthalmicae und zwar der Vv. superiores, seltener der Vv. inferior und begleiten die gleichnämigen Arterien. Ihr intercranielles Stück anastmociert mit dem Sinus sagittalis superior und den Venen der harten Hirnhaut.

III. Zuckerkardl'sche Vene.

Zuckerkandl beschre.bt eine besondere Vene. Sie gehört streng genommen zum System der Ethmoidalvenen. Diese Vene dringt durch die Siebbeinplatte in die Schädelgrube ein und geht entweder in das Venengeflecht des Tractus olfactorius oder direkt in eine stärkere Vene am Orbitallappen über, und in seltenen Fällen erfolgt die Einmündung direkt in den Sinus longitudinalis superior.

IV. Zuckerkandl kcnnte durch Injektion in den oberen Sichelblutleiter folgend: Venen führen :

a) Di: Vene des Stirnbeins, b) einen Teil der Venen der Stirnhöhlenschleimhaut, c) die die Venen im Pfropfe des Foramen coecum, d) die Venen der 
Weichteile und Knochen der äusseren Nase, wenn es sich nur um ein Kind (zumal um ein Neugeborenes) handelt, und e) die Venen der Nasenschleimhaut. Die Nasenschleimhaut einerseit; durch die Verbindung des Sichelblutleiters mit den Ethmoidalvenen und andrerseits durch den Zusammenhang der bereits erwähnten Vene des Orbitallappens mit den ihr selbst zuşehörigen Venen. Der Sinus longitudinalis superior steht durch eine kleine, das Foramen coæcum passierende Vene mit den venösen Geflechten der Schleimhaut des Septi nasium in Zusammenhang. Auf diese Weise können Krankheitserreger, welche bei Verlegungen des Septums nach oben wandern, in den vorderen $T$ eil des Sinus longitudinalis eindringen.

V. Vv. perforantes:

Ausser den bereits beschriebenen Venen besteht noch eine Verbindung zwischen den Venen der Stirnhöhlenschleimhaut und der V. supraorbitalis und dem Sinus falciformis major durch die $V v$. perforantes.

VI. Gurwitsch schreibt, dass in etwa $5 \%$ der Falle aus dem Venennetz der Stirnhöhle eine Vene hervorgeht, welche den Knochen perforiert und sich in die Ursprungsstelle der V. ophthalmica sup. ergiesst.

VII. Venenverbindungen zwischen Kieferhöhle und Orbita.

Auch zwischen Kieferhöhle und Orbita bestehen Venenverbindungen. Das Venengeflecht der Kieferhöhlenschleimhaut entleert sich mittels perforieren der Gefässe an dem Tuberocitas maxillaris, teils in die $\mathrm{V}$ ophthalmo-facialis, teils durch eine, die untere Orbitalwand durchbrechenden Ast in die V. infraorbitalis. Auch hängt das Schleimhautvenengeflecht der Kieferhöhle mit dar V. ophthalmica sup. mittels der V. angularis zusammen (Hajek).

VIII. Venenverbindungen zwischen Sinus cavernosus und Keilbəinhöhlenschleimhautvenen.

Sier und Jakob schreiben, dass für den Verbreitungsweg in der Richtung nach dem Gehirn die Venen zwischen der Schleimhaut der Keilbiinhöhle und dem Sinus cavernosus massgebend sind. Nach den vorliegenden, pathologischanatomischen Befunden zeigt es sich, dass der häufigste Modus, nach welchem die. Keilbeinhöhleneiterungen in die Schädelhöhle sich fortpflanzen, die Durchwanderung der Knochendiploe ist.

IX. Plexus lacrimalis :

Durch den Tränennasengang communicieren die Nasenvenen mit der V. facialis anterior, V. ophthalmica und V. infraorbitalis, dabei wird im Kanal ein 
Schwellgewebe gebildet (Plexus lacrimalis) das den häutigen Tränenkanal für gewöhnlich geschlossen hält.

$\mathrm{X}$. Plexus pterygoideus :

Der Plexus pterygoideus steht durch das Emissarium foraminis laceri und durch das Emissarium foraminis ovalis mit dem Sinus cavernosus in Verbindung. In den Plexus pterygoideus treten ein: die V. maxillaris und mandibularis, sowie die Vv. pterygoideae, massetericae und buccinatoriae. Auch das Venennetz der Kieferhöhlenschleimhaut anastomosiert also mit dem Plexus pterygoideus (Katz, Blumenfeld). Der Abfluss des Plexus pterygoideus erfolgt durch die V. maxillaris interna.

XI. Die rückwärtsabziehenden Venen der Nasenschleimahaut.

a) Aus den hinteren Muschelenden treten oberflächliche Venen hervor, die untereinander und mit den sogleich zu besprechenden tiefen Venen häufig anastomosierend zu den grossen Venen des Schlundiopfes, des Gaumensegels und zu den Venen in der äusseren Nasenschleimhautbekleidung des Keilbeinkörpers ziehen.

b) Tiefliegende Venen begleiten die Äste der A. sphenopalatina durch das Foramen sphenopalatinum und münden in die V. maxillaris interna, eine Wurzel der V. facialis posterior (Zarnico).

XII. Sinus cavernosus :

a) Anatomische Lage des Sinus cavernosus. Sie liegen zu beiden Seiten der Keilbeinkörpers auf den Wurzeln der grossen Fügel und erstrecken sich von der Fissura orbitalis sup. bis zur Spitze des Pars petrosa des Schläfenbeins.

b) Die Verbindung der Sinus cavernosi untereinander:

Die beiden Verbindungen der Sinus cavernosi miteinander, welche an der vorderen und an der hinteren Wand der Sattelgrube sich quer ausdehnen, heissen Sinus intercavernosi anterior et posterior und bilden also mit den Zellblutleiter einen venösen Ring, weldher Sinus circularis genannt wird.

c) Die Verbindung mit den Venen der Grosshirnhemisphäre. Die Venae cerebrales inferiores entleeren ihr Blut bersonders durch die $V$. cerebri media in den Sinus cavernosus, ebenso ergiesst sich der Sinus shenoparietalis in ihn. Dieser Sinus sphenopatrietalis communiciert mit der V. meningea media und nimmt einen Ast von der Dura mater auf.

d) Die Verbindung mit der Augenhöhle:

Wie ich schon genau beschrieben habe, nimmt der Sinus cavernosus die Vv. ophthammicae auf, welche mit der V. facialis durch die V. nasalis in Verbindung 
stehen. In der Aussenwand der Sinus laufen die Nn. oculmotorius, trochlearis, ophthalmicus und opticus zur Orbita.

e) Die Verbindung mit den übrigen Sinus.

Der Sinus cavernosus entleert sein Blut rauptsächlich in die Sinus petrosi. Der Sinus petrosus superior verläuft von hinteren Ende des Sinus cavernosus zum oberen Ende des Sinus sigmoideus. Er verbindet somit den Sinus cavernosus mit dem Sinus sigmoideus und leitet sein Blut zur V. jugularis interna. Der Sinus petrosus inferior ist auch eine andere Verbindung des Sinus cavernosus mit der $V$. jugularis interna. Der Sinus cavernosus varbindet sich mit dem Plexus basilaris, der dem Clivus aufiegt.

f) Die Verbindung mit den Nasenschlimbautvenen:

Die Verbindung zwischen dem Sinus cavernosus und den Venen der Nase und Nasenschleimhaut, habe ich schon oben genau beschrieben.

Aus den beschriebenen Verbindungen der Gefässe der Nase mit den Gefässen der Hirnhäute und den Sinus der dura mater ergibt sich ihr Wichtigkeit für die Fortpflanzung von infektiösen Ertrankungen der Nase und Nebenhöhlen auf die Sinus.

\section{Lymphbahn.}

Die Lymphgefässe der Nase haben eine obəre und eine untere Abflussbahn. Die Obere verläuft zusammen mit den Lymphgefässen der Augenlider zu den Lymphoglandulae paratideae; die untere, von allen Abschnitten der äusseren Nase kommend, verläuft durch d. Lymphoglandnlae buccinatoriae zu den Lymphoglandulae submaxillares.

Die Lymphgefässe der Nasenschleimhaut ziehen teils nach vorn, teils nach hinten. Die Vorderen treten mit den Lymphgefässen der äussern Nase in Verbindung und haben die Lymphoglandulae submaxillares buccinatoriae u d parotideae als regionär: Drüsen. Die hinteren Abflussbahnen sammeln sich in der Nähe des Ostiums pharyngeum tubae auditivae und ziehen zum Teil zu den Lymphoglandulae cervicales profundae, z. $\Gamma$. zur Lymphoglandula retropharyngea lateralis.

Die Regio olfactoria besitzt nun ausser $d: n$ echten Lymphgefässen noch Netze, welche in Analogie zu den Lymphgefässen zu bring $n$ sind. Es sind dieses zarte Lymphräume, welche neben den Scheiden des Nervus olfactorius von den Subarachnoidealräumen aus injizierbar sind. Sie breiten sich nur in der Regio olfactoria des Naseninnern aus und sind als Verbindungen zwischen den Meningen und der Nase anzusehen. 
Der N. opticus besitzt in der Augenhöhle Fortsetzungen der drei Hirnhüllen : Dural, Arachnoideal-und Pialscheide. Das Spatium subdurale und der Subarachnoidealraum erstrecken sich längs des $N$. opticus bis zum Bulbus. Die in ihnen befindliche Lymphflüssigkeit stellt einen direkten Verbindungsweg zwischen dem Bulbus und dem Schädelinnern dar.

\section{Krankengeschichte.}

r. Fall. Septische Thrombose des sinus cavernosus links im Anschluss an Kieferhöhleneiterung rechts.

Anamness: W. I. 20 jähriges Hausmädchen. Patientin bekam vor einigen Tagen Reissen und Ziehen in den Zähnen. Das zahnärtzliche Institut stellte eine Erkrankung der rechten oberen Molarwurzel fest mit beginnendem Wangenabecess.

Befund: Am 6. März 19r5 grosse Schwellung der rechten Kopfseite, insbesondere des rechten Auges, Körpertemperatur $40^{\circ}$.

Operation: Es wird in der rechten Oberkieferseite incisiert, doch ist nicht genau festzustellen, von welcher Stelle aus sich der Abscess gebildet hat. Es entleert sich nicht übermässig viel Eiter.

Verlaaf: 7. III. Die Schwellung ist nicht wesentlich zurückgegangen. 8. III. Die Schwellung geht zurück. Das auge ist frei, Abendtemperatur $40^{\circ}$. I4, III. Temperatur sinkt auf 36,5 und steigt abends auf 41,1 . Schüttelfrost, schlechter Puls. I5. III. Schüttelfrost, Schwellung hinter dem r. Ohr. I8. III. Pdtientin macht einen deutlich septischen Eindruck. Leichter Ikterus, der aber in den nächsten Tagen zurückgeht. Schwellung im Gesicht ist zurückgegangen. Beklopfen der Ohrgend am Schädel nicht schmerzhaft. Urin : wenig Blut, kein Eiweiss. I 9. III. Mundsperre hat nachgelassen. 20. III. Status identisch, nur sensorische Apathie. Ikterus zunehmend. 2I. III. Seit 8 Tagen dauerndes Frösteln, starker Exaphthalmus links. 22. III. Starkes Zittern in der Armmuskulatur, das schon bestanden hat, aber in den letzten Tagen stärker geworden ist. Druck auf beide Jugulares schmerzhaft. Fluktuation nicht fühlbar. Nirgends eine Fluktuation oder Eiterverhaltung. Pupille: Mittelstellung, reagiert auf Licht wenig. Chemosis der unteren Conjunctiva. 23. III. I:terus ist geschwunden, tiefes Atmen spricht für Meningitis. Brustb.fund : spricht für beginnende septische Pneumonie. Am 24. III. 5 Uhr Exitus.

Sektionsbefund: Schweres chronisches Empyem der Ob:rkieferhöhle rechts 
mit Durchbruch in die anstoss $=n d e n$ Weichteile rechts. Linksseitige Thrombose des Sinus cavernosus und des linksseisigen Sinus spenoparietalis. Beginnende eitrige Leptomeningitis an der Fossa Sylvii (s. Fossa cerebri lateralis.). Multiple, meist frische septische Infarkte in beiden Lungen, frische partielle eitrige Pleuritis. Incision am Kiefer, ausgedehnte Phlegmone an der Wangenwand rechts und in der Gegend der Glandulae submaxillaris rechts. Milz leicht septisch. Thrombose im unteren Teil der V. jugularis dextra, Endocarditis verrucosa mitralis.

Dieser eben mitgeteilte Fall ist in verschiedener Beziehung als äusserst interessant zu bezeichnen.

Die bisher in der Literatur mitgeteilten Fälle von Cerzbralkomplikationen nach Kieferhöhleneitrung sind nicht als sicher anzusehen; bei einigen Fällen ist keine gründliche Obduktion vorgenommen worden, und deshalb steht es nicht fest, ob nur die Kieferhöhle beteiligt war und welcher Sinus thrombosiert war.

Bei meinen Fall sind die Anamnese, der Operationsbefund, der Krankheitsbericht und der Obdulitionsbefund so klar und ausführlich, dass sich ein deutliches Bild von dem Krankheitsverlauf gewinnen lässt: der primäre Krankheitsherd ist die r. Kieferhöhle. Alle übrigen Nasenhöhlen sind unbeteiligt geblieben. Die Schwellung der rechten Wange und des rechten Auges sind als Phlegmone oder kollateral entzüdliche Prozesse im Anschluss an den Durchbruch in die anstossenden Weichteile der rechtsseitigen Kieferhöhleneiterung aufzufassen. Am I4. III, trat ein hoher Fieberanstieg ein, nachdem das Fieber in den vorhergehnden Tagen lytisch abgesunken war. Es ist anzunehmen, das der plötzliche Fieberanstieg hervorgerufen wurde durch das Fortschreiten der Infektion nach der Schädelhöhle hin. Der Weg, den die Infektion genommen hat, ist nun sehr eigentümlich: über den Plexus pterygoideus rechts durch die Verbindung zwischen dem Plexus pterygoideus und Sinus cavernosus in das Schäselinnere; von hier sind die Infektionskeime über den Sinus cavernosus und Sinus circularis Ridleyi-ohne diese Sinus zu thrombosieren-nach der anderen Seite gewandert und haben zur Thrombose des linken Sinus cavernosus geführt. Ein ähnlicher Prozesse kann aber auch über die V. ophthalmica entstehen.

Die klinischen Erscheinungen weisen ebenfalls auf die einseitige und zwar die links erfolgte Sinusthrombose hin: Protrusio bulbi links und Schwellung des Augenlides links im Verlauf der Krankheit.

Ein derartiger Fall linksseitiger Sinusthrombose nach rechtsseitiger Kieferhöhleneiterung ist bisher nicht beschrieben worden. Macewen erwähnt, dass eine 
gleichseitige Thrombose über den Sinus circularis Ridleyi nach der anderen Seite sich fortzetzen kann. Er sagt ebenfalls, dass die klinischen Erscheinungen erst auf der gleichen Seite und später auf der anderen hervortreten, ja, dass die Erscheinungen der anderen Seite überwiegen können.

Die im Laufe der Krankheit aufgetretene Schwellung hinter dem rechten Ohr ist wohl als äusserlich bedingt aufzufassen: Schwellung der inficierten prae-und postauriculären Drüsen zusanımen mit einem kollateralen Oedem um den Wangenabecess herum und leichter Stauung in den Gesichts-und Kopfvenen.

Es erweist dieser Fall sicher, dass Oberkieferhöhlenempyem eine tödlich verlaufende Cerebralkomplikation verursachen kann.

2. Fall. Septische Thrombose des Sinus cavernosus im Anschluss an Oberkiferhöhleneiterung.

Anamiese: K. E. 13 jähriger Knabe. Patient hatte angeblich vor 2 Wochen Masern, sonst o. $\mathrm{B}$.

Befund: I9. März 192r. Starke Protrusion des linken Auges mit Schwellung des Augenlides und der Bindehaut. Leichte Benommenheit. Röntgenaufnahme ergibt Vereiterung der rechten Kieferhöhle; allgemeiner Körperbefund o. B.

Operation: am 19.3. wurde der Patient unter allgemeiner Narkose operiert. Schnitt wie bei der Stirnhōhlenoperation in der 1k. Augenbraue. Bei Eröffnung der Orbita entleert sich etwas dünne Flüssigkeit mit Pus, kein Eiterherd; die eröffnete Stirnhöhle ist frei. In der Siebbein-und Keilbeinhöhle findet man keinen Eiter, aber geschwollene Schleimhat. Temperatur abends 40,4 . Puls I60.

Am 20.3. Fieber 40-46,6. Puls 140-165 schwankend. Bewusstlos und Exitus.

Obdutionsbefund: Schleimige Entzündung der Keilbein-und Stirnbeinhöhle, chron. schleimigeitrige Entzündung der Oberkeiferhöhle rechts. Sehr ausgedehnte Orbitalphlegmone links mit ausgedehnter abscedierender Eiterung am oberen Augenlid und der Augenbrauengezend, hochgradige Schwellung des linken Auges. Septische Thrombose der beiderseitigen Sinus cavernosi, der rechtsseitgen V. Ophthalmica und des Sinus sphenoparietalis, sowie der V. cerebri media beiderseits. Multiple Embolien in den Lungenarterien, multiple subpericardiale Blutungen, haemorrhagische Infarkte von stecknadel-bis haselnussgrösse in beiden lungen, eitrige Tracheobronchitis, Oedem und Hyperämie beider Lungen, Hypostase in den Unterlappen, bezinnende fibrinöse Pleuritis. Beginnende eitrige haemorrhagische Meningitis an der Gehirnbasis, eitrige Pachymeningitis in der vorderen und mittleren Schädelsrube, 
sehr stark erhöhter Hirndruck mit Abplattung der Gyri, beginnender Pyocephalus internus. Sehr starke Schwellung des lymphatischen Rachenringes, insbesondere Gaumin-und Zungentonsillen. Septische Milzschwellung mit Beteiligung der Pulpa. Sehr starke Schwellung mit Verfettung und Anaemie des Leberparenchyms.

Auf diesen Fall muss ich ausführlicher eingehen: aus dem Röntgenbefunde, dem Befund der Nasenschleimhaut vor der Operation, aus den Angaben der Operation und Sektion ergibt sich folgendes Bild: I) Die eröffnete Stirnhöhle ist frei, 2) in der Siebbein-und Keilbeinhöhle findet man keinen Eiter, nur geschwollener Schleimhaut, 3) Röntgenaufnahme und Obduktion ergeben Vereiterung der rechten Kieferhöhle, freie linksseitige Kieferhöhle, Thrombose der rechten V. ophthalmica und des Sinus cavernosus beiderseits, 4) Stauungssymptome am linken Auge ohne Thrombose der linken V. ophthalmica.

Ich möchte nun annchmen, uass der primäre Affektionsherd die rechte Kieferhöhle gewesen ist. Die die V. ophthalmica mit den Venen der Kieferhöhlenschleimhaut verbindenden Venengeflechte haben die Infel-tion in die $V$. ophthalmica rechts verpflant und hier zur Thrombose der Vena geführt. Zur Stauung in der rechten Orbita ist es damals nicht gekommen, da bei einer Verlegung der Vene auf einer grösseren Strecke das Blut der Orbita in die Gesichtsvenen abliessen kann (Merkel).

Es ist nun leicht vcrständlich, cass die Thrombose der V. ophthalmica fortschreitend zur Thrombose des r. Cavernosus und im Anschlus daran zur Thrombose des linken Cavernosus geführt hat.

Die Phlegmone des linken Auges mit abscedierender Eiterung am oberen Augenlid, die erst nach der Operation sich voll entwickelt, ist wohl als Metastase aufzufassen; das gestaute, von Blut und Lymphe überfüllte Gewebe der linken Orbita ist als ein locus minoris resistentiae den Infektionserregern gregenüber aufzufassen.

Macewen beschreibt folgende Auffassung; wenn die Affektion von einem Auge auf das andere übergeht, so ist das ein für Thrombose des Sinus cavernosus pathognomonisches Merkmal, doch braucht es bei dem Krankheitsbilde nicht immer vorhanden sein.

Durch meinen, eben angeführten Fall lernt man für die klinlsche Beurteilung des Augensymptomes noch folgendes: Es ist möglich, dass das Auge, über dessen V. ophthalmica die Infektion fortschreitend und zur Thrombose dieser Vene, als auch zu der das Sinus führt, von Stauungssymptomen vollkommen frei bleibt, 
während das andere Auge Stauungssymptome aufweist ohne Thrombose seiner Vene.

3. Fall. Septische Thrombose des Sinus cavernosus und des Sinus longitudinalis superior im Anschluss an Siebbeinzelleneiterung.

Anamnese: A. D. 5 I jährige Frau, wurde bewusstlos ins Krankenhaus eingeliefert. Sie soll lange Zeit an eitriger Sekretion aus der Nase und an vielen Polypen gelitten haben. Seit dem 23.3. 1917 Schwellung der Augenlider.

Befund: Starke Chemosis be:der Augen, starke Protrusio bulbi beiderseits, starke Mydriasis, Lichtschimmer noch vorhanden. Temperatur $39^{\circ}$, kein Schütt $=1$ frost, kochgradige Kopfschmerzen.

Operation: am 27.3. Eröffnung des Siebbeins, Ausräumung des Siebbeins, wobei der Knochen beiderseits sich als sehr morsch erweist. Da die Frau einen asphyktischen Anfa'l bekommt, muss die Operation abgebrcchen werden.

Verlauf: am 28.3. Temperatur 39, $3^{\circ}$, am Abend fallt sie. Patientin ist meistens nicht bei Bewusstsein. Am 7.4. früh 6 Uhr Exitus.

Obduktionbefund: Septische Thrombose des Sinus cavernosus, des Sinus sagittalis superior, des Sinus transversus, des Confluens sinum und der V. jugularis. Eitrige septische Meningitis, besonders an der Basis fleckweise, aber auch an der Grosshirnhemisphäre. Schwere eitrige Rhinitis und schwere eitrige Entzündung der Nebenhöhle der Nase (mit Ausnahme der rechtsseitigen Oberkiefshöhle). Vereiterter kleiner septischer Infarkt in der rechten Lunge, eitrige Bronchitits beiderseits, beginnende eitrige Bronchopneumonie in den rechten Unterappen, beginnende fibrinöse Pleuritis beiders its.

Welche von den Nebenhöhlen hier die Veranlassung zur Thrombose des Sinus gegeben hat, ist nicht zu entschciden. Wahrscheinlich ist aber,- da die Siebbeinzellenentzündung am stärksten war-dass von dort die Infektion im Schädelinnern hervorgerufen worden ist.

Für die Auffassung, dass die Infektion hier von den Siebeinzellen ausgegangen ist, sprechen mehrer Gründe : einmal der klinische Bəfund, Opərations-und Obduktionsbefund und dann das Verhältnis des Venengefelechtes der Siebbeinzellenschleimhaut zu den craniellen Blutleitern (Sinus cavernosus und Sinus longitudinalis superior). Es besteht eine direkte Verbindung mit der V. ophthalmica, die ihrerseits mit ihren craniellen Teil mit dem Sinus sagittalis superior anastomosiert, und auch die V. ophthalnoca führt ihr Blu: in den Sinus cavernosus; dann führen die 
Zuckerkandl'schen Venen durch die Siebbeinplatte in die vordere Schädelgrube, wo sie manchmal direkt in den Sinus sagittalis superior einmünden.

Aber auch die übrigen Nebenhöhlen und die Nase selbst kommen auch als primäre Infektionsherde in Betracht.

4. Fall. Thrombose des Sinus cavernosus in Folge eines Nasenfurunkels.

Obduktionsbefund: S. H. 33 jähriger Mann, am II. 9.21. Exitus. Thrombose des Sinus cavernosus, ausgedehnte Gesichtsphlegmone nach Nasenfurunkel. Entzündung der Nasenschleimhaut im Gebiet der oberen und mittleren Muschel. Empyem der rechtseitigen Keilbeinhöhle. Subduralblutung. Braunes Herz. Haemorrhagi:che, septische Lungeninfarkte in allen Lungenarterien, Bronchopneumonie in den Unterlappen, Lungenödem, entzündliche Hyperaemie des Pharynx, Tracheobronchitis. Anschwellung der septischen Milz. Stauungsgastritis, beginnende saure Erweichung des Magens.

Kritische Bemerkung: es ist hier nicht sicher, ob die Thrombose des Sinus cavernosus durch das Nasenfurunkel cder durch das Empyem der anderen Nebenhöhle hervorgerufen ist. Am wahrscheinlichsten ist, dass als primärer Herd das Furunkel in Betracht kommt (siehe Sktionsbefund), von hier wird die Infektion nach dem Schädelinnern fotgeschritten sein. Welchen Weg die Infektion gegangen ist, lässt sich nicht unterscheiden, da die fehlende Krankengeschichte keine näheren Anahaltspunte gibt.

5. Fall. Thrombose des Sinus cavernosus in Folge eines Furunkels der äusseren Nase.

Anamnese: T. O. 24 jähriger Mann. Mutter tot. Patient hat vor 5 Jahren Masern und Windpocken gehabt, sonst stets gesund gewesen. Im August vorigen Jahres die Gelbsucht und im Dezember Grippe. Vor einem Monat hat Pat. einen Nasenfurunkel mit Nasenbluten gehabt. Seit dem 6.8. 20. Erkälting, Schnupfen, Husten, Temperatur $37,5^{\circ}$.

Fetziges Leiden: Pat. klagte über starke Schmerzen in der linksseitigen Nase. Am I3.8. wurde ein Furunkel links in der Nase festgestellt und Borwasserumschläge gemacht. Die Nasenschwellung ging etwas zurück, ging aber auf die rechte Seite über, und Pat. klagte über sehr starke Schmerzen in der Nasengegend. Temperatur $38,5-39,8^{\circ}$. Am 15.8. machte sich eine leise Schwellung des rechten Augenlides bemerkbar, die stärker wurde. Fieber nält an. 18.8. Pat. sucht die Klinik auf.

Befund: Starker Protrusio bulbi rechts, starke Schwellung des rechten 
oberen Augenlides, die auch auf die Stirnhöhlengegend und den Nasenrücken übergeht. Auch das untere rechte Augenlid ist geschwollen. Rhiniskop.: o. B., Am I9.8. ist das linke obere Augenlid ebenfalls etwas geschwollen, ophthalmoskopische Untersuchg. lks. : o. B., rechts starke Hornhauttrübung (Hypopion).

Operation und Verlauf: In der Orbitia kein Eiter. Eröffnung der Stirnhöhle, auch hier kein Eiter, Siebbinzellen frei. Am 19.8. mittags ist der Pat. noch immer nicht bei Bewusstsein, unruhig, keine Nackensteifigkei ${ }^{+}$. Temperatur 39.3 nicht ausgesprochen. Babinski angedeutet beiders sits. Am 20.8. Nachts unruhig, morgens lässt Pat: Urin unter sich. Linkes Augenlid etwas stärker geschwollen. Pat. ist sehr unruhig, vollkommen bewusstlos, Temperatur 40,3 $3^{\circ}$ Nackensteifigkeit angedeutet, Puls sehr frequent, oft aussetzend.

Obduktionsbefund: Nasenfurunkel, eitrige Thrombophlebitis des Sinus cavernosus, eitrige Pachymeningitis haemorrh., eitrige Leptomeningitis basilaris, “Septicopyaemie." Fitrige Pleuritis. Abscedierungen in den Lungenunterlappen beiderseits, Lungenödem, Bronchitis. Eitrige Pericarditis. Rhinitis Phlegmonosa, Siebbeinhöhleneiterung beiderseits und chronische En zündung der linken Kieferhöhle.

Hier finden wir die Gehirnkomplikation aufgetreten im Anschluss an einen linksseitigen Nasenfurunkel.

In diesem Falle ist der Weg der Infektionskeime wohl folgender gewesen : Von dem Furunkel links ist eine Phlegmone aufyetreten, die sich besonders rechts ausgebreitet hat, von dieser Phlegmone aus ist dann die Infektion des Venengeflechts der rechten Orbitia gekommen (Schwellung der Augrenlider, Hypopion) mit fortschreitender Infektion auf dem Wege der V. ophthalmica ins Gehirn.

Noch ein anderer Weg wäre möglich: Von der Phlegmone können die Keime über die $V$ facialis zum Plexus pterygoideus und von hier in den Sinus cavernosus geleitet worden sein.

6. Fall. Cavernosusthrombose im Anschluss an Keilbeinhöhleneiterung.

Anamnese: N. O. 27 jähriger Mann, stets gesund, als Knabe bekam er einen Hufschlag an den Kopf. Vor etwa 4 Wochen Influenza, nachdem diese abgeheilt war, stellten sich Schmerzen im rechtsseitigen Ohre ein, die täglich stärker wurden; am 3.3. wurde in der Klinik parazentiert, Abfluss von reichlich Eiter. Am 4.3. Aufnahme in die Klinik.

Befund: Pat. klagt über allgemeine Benommenheit, Schmerzen im ganzen Kopf, Fie ber 38,5. Am 5.3. trotz Parazentese keine Erleichterung, Pat. klagt über starken Kopfschmerz in der Stirngegend. Rechtsseitges Lidödem. 


\section{$-14-$}

Operation: am 5.3. Zellen des Prozessus mastoideus rechts zum Teil eingeschmolzen. Granulationen im Prozessus mastoideus und Antrum. Entfernung der Granulationen.

Verlaut : am 5.3. wurde das Lidödem stärker, Protrusio bulbi beiderseits, Chımosis rechts. Am 7.3. auch Chemosis links. Pat: ist benommen, Nasenflügelatmung, Kernig positiv, 4 Uhr nachmittags Exitus.

Obduktionsbefund: Schweres Empyema der rechten Keilbeinhöhle und Kieferhöhle. E trige Thrombose des Sinus cavernosus. Subduraleiterung, frische eitrige Leptomeningitis in der Gegend des Infundibulum und am linken Schläfenpol. Ödem der Lider und der linken Kopfseite. Phlegmone in der Orbitia. Otitis media purulenta dextra. Alter Schädelbruch, durch beide Squamae temporales über die Foramen ovales durch den Türkensattel verlaufend. Dreieckige Bruchlücke am linken Tuber frontale mit starker Impression des hier festverheilten Bruchs'ückes. Vereinzelte bronchopneurnonische Herke in beiden Lungen, beginnende Pleuritis beiderseits. Grosse septische Milz. Mikroskopische Untersuchung des Eiters in den Meningen: Stretococcus longus.

Es ist hier, die Infektion des Schädelinnern auf zwei Arten zu erklären: einmal kann die Infektion von eitriger Mittelohreiterung mit rasch erfogter Einschmelzung des Prozessus mastoideus zur Infektion Bild des Knochenbruches zu Fall 6.

des Schädelinnern geführt haben. Die andere Möglichkeit ist: durch irgend ein Vorkommnis ist der von früher her bestehende Schädelbruch gelockert worden und hat die stark virrulenten Erreger der Keilbeinhöhle durch die Frakturstellen an das Schädekinnere gelangen lassen. Hiermit im Einklaing stehen die Befunde einer frischen

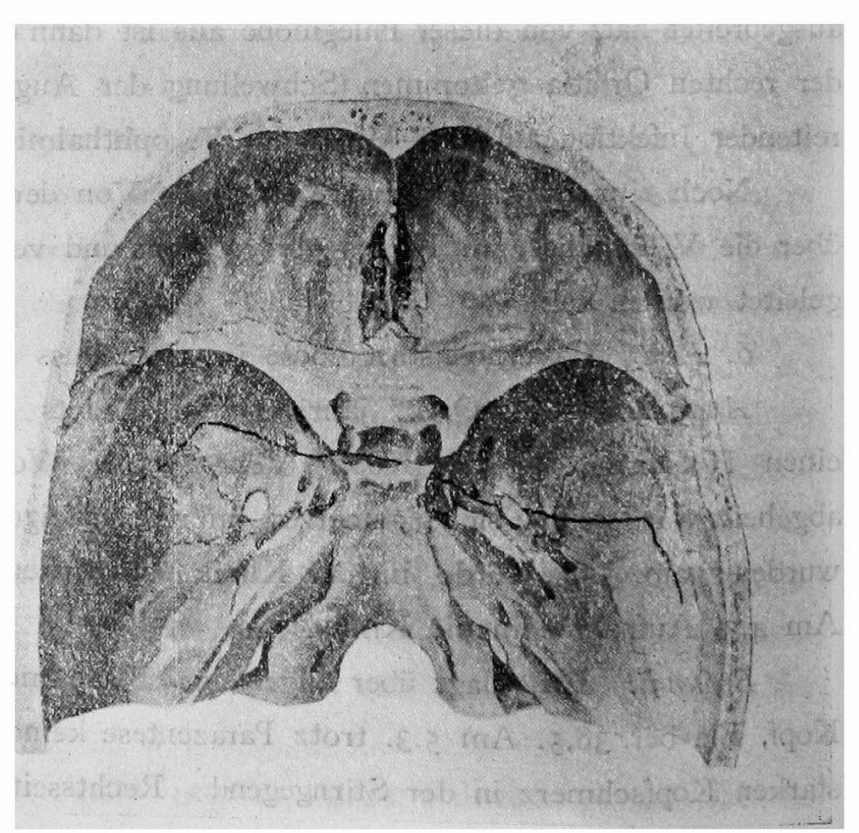


eitrigen Meningitis und der Subduraieiterung im Veraufe der Fraktur und zwar am linksseitigen Schläfenpol und am Infundibulum, während die in Erscheinung getretene Mittelohreiterung rechts lokalisiert war.

7. Fall. Rechtsseitige eitrige Thrombose des Sinus cavernosus im Anschluss an Stirnhöhleneiterung.

Anamnese: R. B. 52 jähriger Mann. Seit 14.3. 1921 ist Pat. wegen der Nase in ärtzlicher Behandlung. Am 18.3. wurde die rechte Kieferhöhle von unteren Nasengang aus geöfinet. Am 20.3. wurde Pat. in bewusstlosem Zustand eingeliefert.

Befund: Oedem der Haut der rechten Stirnseite, Schleimhaut der rechten Nasenseite verschwollen, mit Eiter bedeckt. Nackensteifigkeit, Kerning positiv. Herz, Lunge und Ohren: 0 . B. Temperatur $38,7^{\circ}$

Operation: Eröffnung der rechten Stirnhöhle nach Killian, grosse, mit Eiter gefüllte Stirnhöhle, Krochenwände ohne nachweisbaren Defekt. Resektion des Stirnfortsatzes des rechten Oberkiefers. Resektion des Siebbeins mit dem Chonchotom, Siebbein morsch, Eiter enthaltend. Eröfning der Keilbeinhöhle : o. B.

Verlauf: Pat. nachts sehr unruhig. Am 21.3. ist Fat. noch bewusstlos, reagiert auf keinen Anruf, morgens Temperatur $38,9^{\circ}$, mittags $38,5^{\circ}$, nachts $40, \mathbf{I}$. Am 22.3. um 2 Uhr morgens Exitus.

Obduktionsbefund: Operiertes Stirnhöhlenempyem. Rechtsseitge Vereiterung des Sinus cavernosus. Eitrige Meningitis der Konvexität und der Basis, eitrige Spinalmeningitis mit besonders starker Ansammlung von Eitermassen im Subduralraum, Pyocephalus internus, besonders stark in der 4. Kammer, Vcrwachsung der harten. Hirnhaut mit dem Schädeldach. Eitrige Tracheobronchitis, bronchopneumonische Herde in dem rechten Oberlappen, Induration der Lungenspitzen, Randemphysem der Lungen. Follikuläre eitrige Gaumenmandelentzündung, rechter Follikularabscess, chronische, haemorrhagisch, narbig schrumpfende Gaumenmandelentzündung und chron. nudulärer Rachenkatarrh. Schwellung der Lymphknötchen im Dünn-und Dickdarm, chronische Gastrit's. Pulpaschwellung der Milz.

Der Process befindet sich auf der rechten Seite. Der Hauptinfektions-und Fiterherd ist hier die rechte Stirnhöhle. Zwar sind auch das rechte Siebbein und die rechte Stirnhöhle in den Eiterproscess mit einbegriffen, dcch scheint die Infektion in ihnen an Stärke derjenigen der Stirnhöhle nachzustehen. Von der Stirnhöhle sind die Erreger durch die Vv. perforantes in die V. supraorbitalis, von hier in den hinteren Abschnitt der V. ophthalmica und in den Sinus cavernosus gelangt.

Dieser Fall zeigt keine typischen Stauungserscheinungen am Auge trotz 
Cavernosusthrombose. Durch diesen Fall bemerht man dass auch nasale Cavernosusthrombose kann, ohne Augenerscheiumsgen, entstehen, wie ctogene isolierte Cavernosusthrombose.

8. Fall. Thrombose des Sinus cavernosus nach Operation eines Oberkiefersarkoms.

Anamnese: G. A. 39 jähr. Frau. Im August 1912 hatte Patientin einen lang dauernden starken Schnupfen, damals bemerkte sie zuerst mit dem Finger in der rechten Nase ein kleines blasenartiges Gebilde, das bis Dezember 1912 ungefähr bis zur jetzigen Grösse anwuchs. Es wurde eine Durchleuchtung vom Munde aus mit der elektrischen Lampe gemacht, und der Patientin mitgeteilt, dass die linke Seite frei sei. Seit Ende Dezember 1912 bemerkte die Patientin eine k'eine kirsch grosse Auftreibung unterhalb des linken Auges.

Befund: Am 2. Mai 19r3 Kopfschmerz hin und wieder in der Stirn, Schlai gut, Stuhl unregelmässig. Lungen : Links hinten oben, oberhalb der Spina scapula bei Husten fuchtes, kleinblasiges Rasseln in der Tiefe, linke Seite oben bis unter der Spina scapulae Dämpfung. Reflexe: Patellar sehr gesteigert, Geruchsinn herabgesetzt, Sprache etwas nasal. Auge: Pupillen reagieren normal, links un'erhalb des Auges ist der Proc. zygomaticus kleinkirschgross aufyetrieben. Mundhöhle : oberhalb der oberen Zahnreihe lässt sich die Geschwulst vom 2. linken Schne dezahn bis rechten Eckzahn abtasten, in der Lücke des 2. Prasmolaren oben links gelbliche Schwiele, Fistelgang rot. Nase: Unterhalb des rechten Auges, die ganzee Kieferhöhlen gegend bedeckend, steinharte, nicht druckschmerzhafte Anschwellung, die horizontal $4 \mathrm{~cm}$ misst, Haut verschiebbar und nicht verändert, die rechte untere Muschel ist angeschwollen und so verdrängt, dass das ganze rechte Nasenlumen durch eine graurote harte Platte verlest wird, Septum nach links verdrängt. Postrhinoskopisch: Gerades Septum, leicht glasig verdickte Muschelenden.

Operation: am 23. Mai 1913. Rechtsseitiser Schnitt am Ansatz des Oberkiefers, Verfolgung des Tumors unter dem r. Jochbein. Weiterführung des ersten Schnittes bis auf die linke Seite, Freipräparieren d:s Tumors, der sich vorn völlig scharf umgrenzen lässt und den Eindruck einer Cyste macht. Entfernung eines grossen Teils nach Abmeisseln vom unteren Nasenbodens, wegen sehr starker Blutung Tamponade mit Jodoformgaze darüber 3 Seidennähte.

Am 2. Juni wird von der alten Operationswunde aus nach Entfernung der bsiden unteren Muscheln und der mittleren Muschel rechts am oberen Gaumenboden entlang ge,angen, die Kieferhöhle, die frei von Geschwulstmassen ist, weiter 
geöffnet. Die Knochenpartien werden, soweit Sarkommassen angesetzt hatten ausgiebig mit dem Löffel abgekrazt. Die Wangenschleimhaut wird ebenfalls in der Umgebung des Tumors abgekrazt, von Tumormassen ist nichts mehr fühlbar.

Verlauf: Am 4. Juni klagt Pat. über Husten. Am ro. links hinten in Höhe der Spina scapulae und etwas unterhalb feuchtes Rasseln. Am I5. Temperatur über 38 Grad. Fat. fühlt sich elend und matt, Appetit gering, reichlich eitriger Auswurf. Am I8. keine pleuritischen Erscheinungen. Am 23. sehr starker foetor ex ore, Pat. so elend und schwach, dass sie Essen und Trinken verschüttet und sich nicht aufrecht halten kann. Am 25. Hustenreiz etwas geringer, Puls wechselnd, irregulär, leicht benommen, grosse Schwäche, Sprechen unmöglich. Am 2. Juni I9r3 um 4 Uhr morgens Exitus.

Obduktionshefund: Sarkom des Oberkiefers, Thrombose des Sinus cavernosus, des Sinus sigmoideus und des Bulbus der V. jugularis. Ausgedehnte Osteoporose des ganzen Schädels mit reichlichen Geschwulstmetastasen an beiden Keilbeinflügeln. Metastase des Schädelknochens, insbes. des Keilbeins. Ausräumung und weite Kommunikation der $\mathbf{r}$. Oberki ferhöhle mit der Nase und fistuläre Kommunikation mit der Mundhöhl:. Schwere Gangrän des r. Lungenober-und Unterlappens, Ozdem beider Lungen. Metastasen einiscer Rippen. Ausgedehnte Kalkmetastase beider Nieren.

Es steht nicht fest, welches die direlite Ursache der Thrombose hier ist. Nur das eine ist sicher, dass hier eine Thrombose des Sinus cavernosus und Sinus sigmoideus nach Oberkiefersarkom aufgetreten ist. Es gibt 2 Möglichkeiten : I. dass nach der Operation eine in das Schädelinnere fortschreitende Infektion statt gefunden hat; dann aber auch wäre es denkbar, dass durch dic Knochenmetastasen (insbes. in Keilbein) an den verschiedensten Teilen des Schädelknochens ein Druck auf die Sinus ausgeübt worden ist und es somit $2 \mathrm{u}$ anderen Störungsverhältnissen in ihnen gekommen ist, die dann zur Thrombose geführt haben.

g. Fall. Septische Thrombose des Sinus cavernosus nach $\mathrm{O}_{\text {: }}$ eration eines Oberkief rkrebses.

Anamnese: M. J. 60 jährige Frau. ohne Belang. Jetziges Leiden: Seit 25. Juni 19 Io unter den r. Auge ein kleines warzenähnliches Gebilde, das bald blutet bald geschlossen blieb. Seit I916 vermehrtes Wachstum. Das Knötchen wurde grösser und hart. $1 \frac{3}{2}$ jährige Röntgenbestrahlung erfolglos. Von 19 IO bis I9I5 wurde die Patientin $5 \mathrm{mal}$ operiert. Am 2. März 19\% wurde die Pat. wieder operiert. 
Operation: Auskratzung und Exstirpation des die Augcnhöhle ausfüllenden Tumors; die r. Nasenwand und die Basis cranii zeigen sich durchlöchert. Am 26. März Querschnitt über die Schläfe bis zum äusseren Augenwinkel: Eș wưrden die Knochenreste der äusseren Orbitalwand fortgenommen, darauf liegt die ganze Orbita frei. Das in der Orbita befindliche Carcinom wird mit scharfem Löffel entfernt. Schwere Asphyxie unter zunehmender Schwäche, keine Steifigkeit, Puls regelmässig, verlangsamt, Pat. bleibt bewusstlos trotz Herzmittel. Exitus letalis.

Obduktionsbefund: Oberkieferkrebs. Septische Thrombose des Sinus cavernosus. Diffuse eitrige Meningitis, bes. stark in der $r$. vorderen und mittleren Schädelgrube. Pyocephalus internus geringen Grades. Alte Operationsnarbe der r. Oberkiefergegend und des r. Mundwinkels. Vom Nasenflügel senkrecht aufwärts verlaufende, frische tamponierte Operationswunde der r. Orbitalgegend. Enucleation des Bulbus. Braunes Herz. Hyperaemie der Lungen. Alte indurierte Spitzentuberkulose und Pleuritis.

Es ist wohl anzunehmen, dass in diesem Falle die Infektion post operation aufgetreten ist und zur infektiösen Sinusthrombose bei gleichzeitiger eitriger Meningitis geführt hat.

Man könnte auch annehmen, dass die Thrombose hier hervorgerufen wurde durch die mit dem Garcinom einhergehende Kachexie. Es wäre dann einer der seltenen Falle von eitriger Meningitis als Folgezustand einer marantischen Thrombose. Die Möglickeit dieses Vorkommens gibt auch Maseven zu.

IO. Fall. Septishe Thrombose des Sinus longitudinalis superior im Anschluss an Stirnhöhlenempyem.

Anamnese: R. I. I2 jähriges Mädchen. Die Mutter der Patientin ist an Tuberkulose gestorben. Das Mädchen hatte im 2. Jahre Rhachitis, im 6. Jahre Windpocken. Seit Anfang des Aprils I9ro Augenentzündung. In der Augenklinik wird Tenonitis festgestellt. Am 7. April wurde an der Nasenwurzel ein schmerzhafter, fluktuierender Tumor bemerkt. Am 8. April Eröffnung der Geschwulst in der Chirurgischen Klinik. Fieber 38-40 Grad. Am II. April trat eine starke Schmerzhaftigkeit und Schwellung hinter dem r. Ohr auf, ferner etwas Nackensteifigkeit. Nachträglich wird von der Mutter angegeben, dass das Mädchen oft und lange an Kopfschmerzen gelitten hat.

Befund: Das Mädchen wird in etwas benommenem Zustande gebracht, Temperatur 40,I Grad. Das Mädchen ist sehr empfindlich, so dass jede Berührung 
schmerzhaft ist. Ueber dem r. Auge eine frische Incisionswunde. Hinter dem r. Ohr eine schmerzhafte Stelle, die leicht fluktuiert.

Operation: I I. April. Die r. Stirnhöhle wird geöffnet, die mit Eiter gänzlich ausgefüllt ist. Aus einer Kommunikation zwischen Schädel, Orbita und Nase entleert sich ein Tropfen Eiter makroskopisch. Ferner wird hinter dem r. Ohr ein Bogenschnitt geführt; man trifft dabei kaum Eiter an, wohl aber viel thrombosierte Venen. I2. April. das Mädchen ist nachts sehr unruhig gewesen, Temperatur 39,9-41,2 Grad. Abends wird der Schnitt hinter dem Ohr nach dem Kiefergelenk zu verlängert, jedoch trifft man auf keinen Eiterherd. Starker Durchfall. I3. April : Temperatur ist auf 39 Grad gefallen, gegen Abend 38,2. Die Durchfälle halten an. Am I4. April nachts sehr unruhig, sehr verfallen, Temperatur 36,4 Grad, um I I Uhr nachts Exitus.

Obduktionsbefund: Doppelseitiges Stirnhöhlenempyem (r. operativ geöffnet). Septische Thrombophlebitis des Sinus longitudinalis sup. Oedem und Hyperaemie der Pia und des Gehirns. Beginnende Phlegmone an der r. Halsseite. Septische Lungeninfarkte. Subpleurale und subpericardiale Hämorrhagien. Milzschwellung. Katarrhalische Enteritis. Schädeldach : Dura stark gespannt, an ihrer Aussenfläche, entsprechend der Spitze des r. Stirnlappens, ein to pfennigstückgrosser, grünlichgelber, ziemlich fest haftender Belag. Der gegenüber liegende Knochen ist nicht auffalllg' verändert, dagegen ist die Dura in der ganzen Umgebung auffallend saftreich. 'Im Längssinus finden sich im vordersten Abschnitt, der eben beschriebener Stelle unmittelbar benachbart ist, gelblich-graue, teils zerfliessliche, teils der Wand fester anhaftende Massen. Weiter hinten enthält der Sinus schmutzigrote Cruorgerinnsel, teils geblich-graue bis bräunlich-graue, teils mehr gallertartige, teils zerfliessende Massen. Innenfläche der Dura spiegelnd. Pia und Gehirn ziemlich saftreich, sonst ohne Veränderungen. An der basalen Dura bes. in der hinteren Schädelgrube ziemlich zahlreiche, stecknadelkopf-bis linsengrosse, rote Fleckchen, die sich nicht abspülen lassen. Nach Abziehen der Dura ist an den Furchen der Basis nichts auffälliges zu sehen. Beim Aufmeisseln der r. Orbita quillt sofort gelbgraue, dicke Flüssigkeit hervor. Die r. Stirnhöhle entleert blutige, schleimige Massen.

Hier haben wir einen Fall von Stirnhöhlenempyem mit nachfolgenden Gehirnkomplikationen : Kopfschmerz, Nackensteifigkeit, Or'italvereiterung, Abscessbildung an der Nasenwurzel, allgemeine Sepsis mit Thrombosierung der Venen hinter dem 
Ohr mit Lungeninfarkten und septischer Milz. Die Thrombose des Sinus longitud. sup. wird auf dem Wege über die Vv. perforantes statt gefunden haben.

Es wäre auch denkbar, dass die Infektion der Blutleiter durch die Infizierung der Orbita vor sich gegargen ist, doch die Veränderung des Schädelknochens an der Stelle der Stirnhöhle, das Anschwellen der Orbita, sowie die Angabe der Mutter über das lange Bestehen der Stirnhöhleneiterung lassen die Infektion der Stirnhöhle sicher als primäre erscheinen.

II. Fall. Thrombose des Sinus longitudinalis sup. im Anschluss an Stirnhöhlenempyem.

Anamnese: 20 jähriger Mann. Vorher nie ernstlich krank gewesen sein. Am 29. September I 92 I bemerlte Pat. heftige Kopfschmerzen, meldete sich krank und wurde am selben Tage dem Lazarett überwiesen.

Befund: Mittelkräftiger Mann in ausreichendem Ernährungszustand, sichtbare Schleimhäute gut durchblutet, Temperatur 39,4 Grad, Puls 97 Schläge in der Minute, regelmässig, kräftig. Rachen: hintere Rachenwand gerötet. Nasenwurzel und Stirngegend bis 2 fingerbreit über den Augenbrauen oedematös geschwollen. L. Auge fast zugeschwollen. Kein Exophtalmus, kein Schielen. Starke Druckund Klopfemphfindlichkeit der Stirnhöhlengegend. Herz, Lunge und Bauch ohne Befund.

Verlauf: 30. Sept, kein Rückgang der Erscheinungen. Am r. Oktober hat sich die oedematöse Schwellung bis zur Haargrenze ausgebreitet, kein Exophtalmus, kein Schielen. Wegen des akuten, fortschreitenden Prozesses wurde Fat, zur operativen Behandlung in die Nasenklinik verlegt.

Befund: 2. Okt. I92 I starke Schwellung der 1. Stirnseite bis herauf zum Schädeldach. Pus in der Nase nicht vorhanden. Hohes Fieber.

Operation: Eröffnung der 1. Stirnhöhle, die völlig mit Eiter erfüllt ist. Die erkrankten Teile des Knochens an der Rückwand werden entfernt und ein Hirnabscess freigelegt. Ausräumung des Siebbeins.

Verlauf: 3. Okt. Fieber dauernd hoch, Pat. ist benommen, unruhig, Nackensteifigkeit. Lumbalpunktion: Druck $350 \mathrm{~mm}$, im Liquor viele Leukocyten, bes. polymorphkernige, wenige Lymphozyten und Erythrozyten. Liquor etwas trüb, flockig (Meningitis purulenta). In weiterem Verlauf n:ehrfache Krämpfe, Nackenstarre, Benommenheit. Temperatur 4I,I Grad und zunehmende Herzschwäche. Am 4. Oktober um I Uhr nachts Exitus letalis.

Obduktionsbefund: Operierte Stirnhöhleneiterung. Eitrige Leptomeningitis 
beider Frontallappen. Thrombose des Sinus longitud. sup. Fibrinös-eitrige Rhinitis beiderseits. Chronischer, stark indurierender, cavernöser, taubeneigrosser, tuberkulöser Herd der linken Lungenspitze, an andern Stellen Oedem, kleine Blutungen und Entzündungen der Pleurablätter 1. Pulpa-und Follikelschwellung der Milz (189g).

Dieser Fall liegt wie der vorige: Der primäre Entzündungsherd ist die Stirnhöhle. Die Infektion kann auf 2 Wegen an den Sinus longitud. sup und in ihn hinein gelangt sein: Einmal direkt durch die Knocheneiterung und Gehirnabscessbildung, und dann auf dem Wege der Vv. perforantes.

$\mathrm{Zu}$ bemerken ist noch, dass im Verlauf der Krankheit Krämpfe auftraten. Diese Krämpfe, die man als Symptome einer Thrombose des Sinus longitudinalis sup. und der Venen der motorischen Rinderzentren auffasst, treten häufiger bei Kindern, als, bei Erwachsenen auf.

12. Fall. Thrombose des Sinus longitud. sup. im Anschluss an Stirnhöhlenempyem.

Obduktionsbefund: M. B. 20 jähriger Mann, am 3. Januar 1920 Exitus. Empyem beider Stirnhöhlen. Septische Thrombose des vorderen Abschnittes des Sin. longit. sup. (die übrigen Sinus und die V. jugul. frei). Pachymeningitis externa et interna. Beginnende eitrige Hirnhautentzündung der medialen Fläche des $r$. Stirnhirns neben Thrombophlebitis zweier Piavenen, starke Hyperämie des Gehirns. Siebbeinzellen, Keilbeinhöhlen frei von Veränderungen. Doppelseitige fibrinös-eitrige Pleuritis infolge zahlreicher metastatischer, septisch-hämorrhagischer Infarkte und Abscesse beider Lungen. Confluierende Bronchopneumonie und Oedem der Unterlappen. Operationsschnitt längs der Augenbraue und an der Nase entlang. Indurierende Phlegmone der Weichteile der linken Augenhöhle. Hyperaemie des Magen-und Darmkanals. Gering Hyperplasie der Milzpulpa.

Durch den Sektionsbefund allein ist hier bewiesen, dass als Primärinfektionssitz die Stirnhöhle anzusehen ist. Der Weg der Infektion wird derselbe gewesen sein, wie in den vcrher beschriebenen Fällen.

13. Fall. Thrombose des Sinus longitud. sup. im Anschluss an Stirnhöhlenentzündung.

Obduktionsbefund: H. A. 39 jähriger Mann, am 12. Dezember 1916 Exitus. Septische Thrombose des hintersten Abschnittes des Sinus longitud. sup. und des Confluens sinum. Frische handtellergrosse Trepanation mit Gehirnprolaps in der Gegend der r. Zentralwindungen. Eitrig-jauchige Entzündung der beiderseitigen Stirnhöhlen und Oberkieferhöhlen. Todesursaehe: Meningitis nach neiven Nasen- 
höhleneiterung. Schwere eitrig-jauchige Lepto-und Pachymeningitis über beiden Grosshirnhemisphären; geringe beginnende, bes. vorn starke, intermeningeale Blutungen, bes. in der Nähe der Falx.

Die Trepanationsstelle bəi diesem Fall wird nicht auf den richtigen Weg führen bei der Suche nach dem Infektionsherd. Es ist anzunehmen, dass unbestimmte Symptome von Seiten des Kranken bestanden haben, so dass die Chirurgische Klinik mit der Trepanation wohl nur eine Hirndruekentlastung vornehmen wollte.

Die Thrombose des Sinus longitud. sup. lässt eher rückschauend vermuten, dass hier eine Stirnhöhlenerkrarkung als Infektion vorgelegen hat.

Bei der Thrombose des Sinus longitudin. nach Nasenerkrankung kommt es meist zur Verlegung im vorderen Abschnitt des Blutleiters, aber bei diesem Fall beobachtet man die Thrombose am hintersten Abschnitt.

\section{Statistischer Zusammenhang der Sinusthrombosen im Anschluss an Nasenerkrankungen.}

Wie ich schon auf der Tafel bai Fällen der otogenen Cavernosusthrombose geschrieben habe, zeigt die Statistik kurz noch einmal: bei 18763 Obduktionen der Jahre 1909 bis 1923 habe ich an Gehirnkomplikationen nach Nasenerkrankungen 77 Fälle und nach Ohrerkrankungen 352 Fälle gefunden. Von den 77 Fallen nach Nasenerkrankuneen entfallen 9 auf Thrombose des Sinus cavernosus, 4 auf Thrombose des Sinus sigmoideus und 5 ruf die des Sinus longitud. sup. Die restlichen Fälle waren Gehirnkomplikationen ohne Thrombose. Von den 352 Fällen nach Ohrerkrankungen entfallen ig auf die Cavernosusthrombose, 70 auf Sigmoideusthrombose und 22 auf die Longitudinalthrombose.

Es zeigt sich ein interessantes Ergebnis:

I. Das Verhältnis der Thrombose des Sinus longitud. sup. bei Ohr-und bei Nasenerkrankungen zu den bei diesen Erkrankungen vorkommenden Gehirn'omplikationen überhaupt beträgt ca $6 \%$.

2. Das Verhäitnis der Thrombose des Sinus cavernosus zu den Gehirnkomplikationen nach Nasenerkrankungen beträgt ca $12 \%$.

3. Das Verhältnis der Cavernosusthrombose zu den Gehirnkomplikationen nach Ohrerkrankungen beträgt ca $5 \%$. 


\section{Pathogenese.}

Die Cerebralaffektionen im Anschluss an Nasenerkrankungen gehören zu den gefährlichsten Komplikationen. Sie betreffen Veränderungen der harten und weichen Hirnhaut (Extra-und Intraduralabscess und Meningitis) oder die Hirnsubstanz (Hirnabscess). Manchmal pflanzt sich die Entzündung auf dem Wege der Thrombophlebitis fort, und es kommt zur Sinusthrombose. Bevor ich aber darauf näher eingehe, möchte ich einiges über die Pathogenese der Cerebralaffektionen im Gefolge von Nasenerlkrankungen sagen.

\section{Infeltiöse Ursachen.}

a) Eine infektiöse Entzündung der Nasenebenhöhlen (der Oberkiefer-, Stirnoder Keilbeinhöhlen und des Siebbeinlabyrintes) kann durch akuten Schnupfen, bei Influenza, Scharlach, Masern, Thyphus, Pneumonie etc. erfolgen; und aus der akuten Entzündung entsteht die chronische, namentlich, wenn es zu Sekretretention kommt, ferner auch häufiger bei Wiederholung akuter Sinusitis und starker Virulenz der Bakterien. Sie können sich ferner im Anschluss an osteomyelitische und syphilitische Knochenprozesse entwickeln. Solche infektiösen Entzündungen der Nasennebenhöhlen können zuweilen die Knochenwand zerstören und Eiter direkt in das Schädelinnere dringen lassen, oder in die Weichteile durchbrechen, und dann können die Entzündungskeime auf dem Umwege einer Phlegmone mit anschliessender Phlebitis in die Blutleiter der Schädelhöhle gelangen.

b) Ausserdem sind es in der Nase und den Nebenhöhlen gewöhnlich Furunkel und Phelgmonen der äusseren Nase, Traumen des Nasendaches, Frakturen der Knochenwand der Nebenhöhlen, Verletzungen mit Wundinfektion, Folgezustände tuberkulöser und syphilitischer Ulcera auf der Membrana pituitaria und maligne Tumoren, welche direkt oder indirekt zur Hirnkomplikation führen.

Diese Komplikationen werden hervorgerufen durch die entweder im Eiter verschleppten oder auf dem Blut-und Lymphwege eingewanderten Bakterien. Es zeigen sich alle Arten von Frregern daran beteiligt : Streptococcen, Staphylococcen, Pneumococcen, Influenzabazillen, Diphteriebazillen und in seltenen Fällen Kolibakterien und Meningococcen.

2. Nicht infektiöse Ursachen.

Die bisher beschriebenen pathogenetischen Grundlagen für eine Sinus- 
thrombose bauten sich als sekundäre Erscheinungen auf einer Infektion auf. Es sind hier noch die Thrombosen zu erwähnen, die hervorgerufen werden durch Gefässwandschädigungen oder Störungen der Blutströmung an Ort und Stelle.

Man könnte sie primäre Thrombose des Sinus nennen; doch wäre dann der Ausdruck "primär" nicht rein gebraucht, da ja schliesslich die Wandveränderungen und Strömungsänderungen auch nur Folgeerscheinungen anderer krankhafter Vorgänge im Körper sind. Derartige Thrombosen sehen wir bei Arteriosklerose, bei Diabetes melitus und bei Kachexie durch Geschwülste; es ist daher auch bei Nasentumoren eine Thrombose des Sinus denkbar. Zur Thrombose kann es ebenfalls kommen bei Geschwülsten der Knochenwand, der Meningen oder des Gehirns, die durch den von einem Tumor ausgeübten Druck den Blutleiter obliterieren.

\section{Disponierende Momente.}

a) Hier spielen eine wichtige Rolle Defektbildungen in d:r Knochenwand der Nebenhöhlen. Bei der Stirnhöhle kommen solche vor sowohl in der an die Schädelhöhle grenzenden Lamelle des Proc. orbitalis, wie auch an der Orbitalplatte. In dem letzten Falle kommt es zunächst zur Bildung eines Orbitalabscesses mit nachfolgender, fortschritender Entzündung oder zur Thrombophlebitis ophtalica. Bei der Defektbilung der hinteren Wand der Stirnhöhle greift die Entzündung direkt auf die Hirnhaut über. Bei dem Siebbein gibt es Defekte in der Lamina papyracea und Lamina cribrosa. Durch die Lamina papyracea hindurch entsteht zunächst wieder ein Orbitalabscess, der fortgeleitet wird, wie es bei der Stirnhöhle beschrieben wurde: Die Keilbeinhöhle zeigt Defekte in den oberen seitlichen Winkeln, wodurch die Eiterung direkt auf das Schädelinnere übergehen kann.

b) Alte, nicht vollständig verheilte Risse von früheren Frakturen der Knochenwände disponieren ebenialls für eine von der Nase aus fortschreitende Infektion des Schädelinnern.

c) Weitere disponierend: Momente können in dem absoluten oder relativen Verschluss der Ausführungsgänge der Nasennebenhöhlen erblickt werden.

d) Als ein prädisponierendes Moment zur Thrombosierung kommen unter gewissen Umständen Veränderungen in dem normalen Strömungsverhältnis vor. Die Verengerung des Venenlumens durch Druck eines Prozesses disponiert Entstehung der Thrombose; aber duch Druck allein kommt eine Thrombose nicht zustande; sie setzt immer die toxischen Einwirkungen der entzündlichen Vorgänge der Aussenfläche des Sinus oder die Veränderung der Blutbeschaffenheit voraus. 
4. Infektionswege.

a) Die Eiterung der Nasennebenhöhlen kann sich dadurch einen Weg nach dem Schädelinnern bahnen, dass die Schleimhäute zunnächst zerstört, ein subperiostaler Abscess gebildet und dann der Knochen eingeschmolzen wird. (Direkte Fortpflanzung in der Gewebskontinuität). Auf der cerebralen Seite des Knochens kann es dann zur Bildung der Intraduralabscesse kommen, die wied -r Meningitis, Hirnabscess oder Sinusthrombose zur Folge haben können.

Diese Thrombosierung geht vor sich, indem zunächst noch al'mählicher Erkrankung der Venenwand in Form einer Lymphangitis das Blut durch diffundierenden Gewebssaft zur Gerinnung gebracht wird und erst nachträslich die Infektion durch Bakterien stattfindet.

b) Ausser dieser direkten Fortpflanzung der Infektion und der Gewebskontinuität kommen als Wege für die Fortpflanzung die Venen in Btracht. Welche Venen dabei eine Rolle spielın, habe ich im anatomischen Teile bereits genau beschrieben. Namentlich eine Thromhophlebitis, die in der Schleimhaut einer Nasenebenhöhle entstanden ist, kann durch die Venen cerebralwärts fortgeleitet werden, so dass es zur Thrombose der V. ophtalmica und weiter zur Thrombose des Sinus cavernosus oder direkt zur Thrombose des Sinus longitudinalis superior kommen kann. Ausser auf diesem Wege kann die Infektion auch durch den Plexus pterigoideus zum Sinus cavernosus fortschreiten.

c) Ferner kann sich die Entzündung durch Lymphbahnen nach dem Gehirn hin fortsetzen; ebenso können die Nervenscheiden die Wege bilden, auf denen die Mikroorganismen einwandern.

d) Ausserdem kann das Gehirn mit seinen Häuten angegriffen oder zerstört werden durch die Bildung sogenannter regionärer Metastasen. Bei diesen Fällen ist der an das Cavum cranii angrenzende Knochen nicht zerstört. Die Stelle, wo der Prozess durchgedrungen ist, zeigt dann nur eine cirkumskripte Verfärbung des Knochens und der Dura. In andern Fällen kommt es deutlich zur Bildung eines Extra-und Intraduralabscesses, wobei der darunter liegende Knochen nur geringfügig verfärbt ist.

\section{Symptome.}

Die Thrombose des Sinus cavernosus und des Sinus longitudinalis superior ist ganz selten als einziger Befund bei einer Nasenerkrankung vorhanden. Zumeist 
ist die Thrombose vergesellschaftet mit einer Meningitis, einem Hirnabscess, oder mit Lungen-und Darmkomplikationen oder mit einer anderen Folgererscheinung der allgemeinen Sepsis.-Es ist daher schwer, ein bestimmtes, für die Sinusthrombose charakteristisches Krankheitsbild zu zeichnen.

a) Symptome der Thrombose des Sinus longitudinalis sbperior. Hajek hält nur die Schmerzen der Scheitelgegend und pyaemisches Fieber für einigermassen charakteristisch. Killian erwähnt mehrere Symptome: lebhafte Stirnhöhlenschmerzen, Fieber, Schmerzen in der Scheitelgegend, bei Zerfall des Thrombus Schüttelfrost, remittierendes Fieber. Maceven schreibt, es gäbe keine pathognomischen Merkmale: Mit einiger Sicherheit sprechen folgende Begleiterscheinungen für eine Thrombose: Oedem der Kopfhaut, starke Erweiterung der Venen in der Parietal-, Occipitalund zuweilen auch Frontalgegend, mitunter ein leichter Grad von Schielen, Epistaxis, Abscesse auf der Aussenseite des Schädels, Krämpfe mit nachfolgender Lähmung und bei Kindern hochgradige oedematöse Schwellung der Kopfhaut über der vorderen Fontanelle. Auf meine Fälle bezugnehmend habe ich noch $z u$ bemerken : ausser den oben zitierten Symptomen tritt als Merkmal der Thrombose eine starke Anschwellung und Schmerzhaftigkeit hinter dem Ohre auf ohne Mittelohreiterung und ohne Thrombose des Sinus sigmoideus.

b) Symptome der Thrombose des Sinus cavernosus. Maceven gibt eine ausführliche Schilderung: er unterscheidet 2 Symptomengruppen : die eine beruht auf dem Verschluss des Blutleiters und auf der dadurch bedingten Rückwirkung auf das Auge und das retrobulbäre Zellgewebe ; es entstehen Oedem der Lider, Chemosis, Exophthalmus und Papillitis optica. Die 2. Gruppe der Symptome sind Lähmungserscheinungen, die durch Druck auf die der Orbita benachbarten Nerven hervorgerufen werden: Ptosis, Strabismus, Pupillenstörung und Paresen der Augenmuskeln. Alle diese Symptome können in ganz verschiedener Intensität auftreten.

I) Zu dem hier angeführten muss ich betonen, dass diese Stauungs-und Lähmungserscheinungen sehr variabel und äusseast unzuverlässig sind. Es kommt sogar vor, dass keines der sogenannten Augensymptome vorhanden ist. Auch bei meinen Fällen der otogenen, isolierten Cavernosusthrombose sind solche Ericheinungen nicht aufgetreten. Ich habe daruber in September 1924 berichtet.

2) Durch meinen Fall lernt man für die klinische Beurteilung der Augensymptome noch folgendes: bei nur einseitiger Nasennebenhöhleneiterung kann aus der fortschreitenden Infektion sich eine Thrombose des Sinus cavernosus der anderen Seite entwickeln, ohne dass eine Thrombose des Sinus cavernhsus derselben Seite 
zu bestehen braucht. Ebenso können sich Augenkomplikationen (Stauungs-und Lähmungserscheinungen) auf der andern Seite ausbilden.

3) Ausserdem habe ich einen Fall beobachtet, bei dem das Auge, über dessen V. ophthalmica die Infektion fortgeschritten ist und zur Thrombose dieser Vene wie auch des Sinus cavernosus geführt hat, von Stauungs-und Lähmungserscheinungen vollkommen frei geblieben ist, während das andere Auge Stauungs-und Lähmungserscheinungen ohne Thrombose der V. ophthalmica dieser Seite aufwies.

c) Allgemeine Symptome bei der Sinusthrombose und Komplikationen.

Als allgemeine Symptome bei der Sinusthrombose treten auf: 1. Kopfschmerz in der Gegend des primären Herdes und der erkrankten Blutleiter oder in deren Umgebung; 2. häufiges Erbrechen; 3. plötzlicher Anstieg der Temperatur (38-4I Grad); 4. schwankender kleiner Puls; 5. Schüttelfrost mit profusen Schweissen.

Hierzu kommen die zahlreichen Symptome der oft gleichzeitig bestehenden allgemeinen Sepsis : Lungenkomplikationen (Dyspnoe, Husten, Schmerzen der Lungen, foetider Auswurf, foetor ex ore), Verdauungskomplikationen (trockene, pilzige Zunge, Foetor ex ore, Anorexie, typhöse Symptome), Leberkomplikationen (Schmerzen, Ikterus), Milzanschwellung und septische Unterhautblutungen. Als gleichzeitig bestehende Komplikationen kommen weiter in Betracht: Hirnabscess und Meningitis (Kopfschmerz, heftig oft in der Stirngegend, häufges Erbrechen, Temperatur dauernd hoch, manchmal Schüttelfrost, Erregbarkeit, Hypersensibilität, Unruhe, tonische und klonische Krämpfe des Gesichtes und der oberen Extremitätenmuskulatur, Schielen, Coma etc.).

d) Differentialdialnose.

a) Hirnabscess.

Das Krankheitsbild eines Hirnabscesses zeigt folgende Zusammensetzung : langsamer Puls, normale Temperatur, Krämpfe im Initial-und Endostadium, Erbrechen selten (bei Kleinhirnabscess häufig), Reflexe unsicher, Gesichtsausdruck schwer leidend. Dagegen bei Sinusthrombose zeigt sich beschleunigter, schwacher Puls, hohes Fieber mit Remission, Krämpfe und Erbrechen. Beiden Erkrankungen gemeinsam sind verlangsamte Hirntätigkeit, falscht oder verwirrte Antworten, Benommenheit.

b) Extraduralabscesse.

Sie könen verschiedene Folgeerscheinungen haben; sie können Sinusthrombose veranlassen, Hirndruck und lokale Herdsymptome zur Folge haben und anfangs auch vollkommen symptomlos verlaufen. 
e) Meningitis.

Ihre Symptome sind gewöhnlich so deutlich, dass sie nicht zu übersehen sind. Sicheren Aufschluss über eine etwa zweifelhaften Hirnhautprozess gibt die Untersuchung des Liquor cerebro-spinalis.

d) Nicht rhinogene Krankheiten mit ähnlichen Symptomen.

Bei solchen nicht rhinogenen Erkrankungen, wie Thyphus abdominalis, septische Endocarditis, Malaria, Miliartuberlkulose, akute Pneumonie, puerperale Sepsis und Erysipelas etc., ist*es nötig, durch Anamnese und genaue Nasenuntersuchung eine Beteiligung der Nase und der Nasenebenhöhlen auszuschliessen.

e) Thrombose des Sinus sigmoideus.

Wenn b.i Thrombose des Sinus sigmoideus Oedem vorhanden ist, dann handelt es sich um eine geringe Schwellung, welche hinter der Ohrmuschel auftritt und sich bis zur hinteren Seite des Warzenfortsatzes und abwärts etwas über seine Spitze hinaus ausbreitet. Häufig ist die Percussion des Warzenfortsatzes schmerzhaft. Wenn bei der Operation die Sinuswand gesund und normal aussehend gefunden wird, so kann men meistens eine Thrombose sofort ausschliessen.

\section{Ergebnisse.}

I. Das Verhältnis der Thrombose des Sinus longitudinalis superior bei Nasenerkrankungen zu den bei dieszn Erkrankungen vorkommenden Gehirnkomplikationen überhaupt beträgt ca $6 \%$. Das Verhältnis der Thrombose des Sinus cavernosus zu den Gehirnkomplikationen nach Nasenerkrankungen betr gt ca $12 \%$.

2. Nach Kieferhöhleneiterung kann eine tödliche Sinusthrombose eintreten. Dieses Ergebnis ist nicht so solten, wie man es nach der anatomischen Tage bei oberflächlicher Betrachtung annehmen könnte.

3. Alte, nicht vollständig verheilte Risse von früheren Frakturen dər Knochenwände der Nebenhöhlen disponieren für eine von der Nase aus fortschreitende Infektion des Sinus.

4. Stauungs-und Lähmungserscheinungen auf das Auge bei Cavernosusthrombose sind sehr variabel und äusserst unzuverlässig. Es kommt sigar vor, dass dicse sogenannten Augensymptome auch bei nasale Cavernosus thrombose nicht auftreten können, wie bei otcgenen, isolierten Cavernosusthrombose.

5. Bei nur einseitiger Nasennebenhöhleneiterung kann aus der fortschreitenden Infektion sich eine Thrombose des Sinus cavernosus der anderen Seite entwickeln, 
ohne dass eine Thrombose des Sinus Cavernosus derselben Seite zu bestehen braucht. Ebenso können sich Augenkomplikationen (Stauungs-und Lähmungserscheinungen) auf der anderen Seite ausbilden.

6. Es ist möglich, dass Auge über dessen V. ophthalmica die Infektion fortschreitet und zur Thrombose dieser Vene wie auch des Sinus cavernosus führt, von Stauungs-und Lähmungserscheinungen vollkommen frei bleibt, während das andere Auge Stauungssymptome und Lähmungserscheinungen aufweist ohne Thrombose der $\mathrm{V}$ ophthalmica seiner Seite.

7. Schmerzhaftigkeit und Anschwellung der Regio mastoidea ohne Mittelohreiterung, ohne Mastoiditis oder ohne Sigmoideusthrombose kommt zuweilen nicht bloss bei Longitudinalisthrombose nach Nasenerkrankungen, sondern auch bei rhinogener Cavernosusthrombose vor; denn diese Symptome sind Zeichen von Stauungssymptomen oder septischen Erscheinungen an den Venen und Lymphbahnen des Kopfes.

Zum Schluss möchte ich an dieser Stelle Herrn Professor Dr. Lubarsch, Herrn Professor Dr. Passow und Herrn Professor Dr. von Eicken für die Erlaubnis, das für meinen $Z$ weck geeignete Material zu verwerten, meinen herzlichsten Dank aussprechen.

\section{Literatur-Verzeichnis.}

Hajek, Nebenhöhlen der Nase. 1915.

Znckerkand1, Anatomie der Nasenhöhle, 1893.

Rauber-Kopseh, Lehrbuch d. Anat. X. Aufl. (III) 1914.

Zarnico, D. Krankheiten der Nase und d. Nasenrachens, III. Auf. 1910.

Maceven, Infekt. eitr. Erkrankg. d. Gehirns u. Rückenmarks 1898.

Merkel, D. Anat. d. Menschen 1913.

Sier u. Jakob, Recherhces anatom. cliniques et operat. sur les fosses nasales et leurs sinus 1901.

Killian, D. Thrombophleb. d. ob. Längsblutleiters a. Entzdg. d. Stirnhöhlenschleimhaut. Verein Süddeutsch. Laryngol 1900.

Finlay, Arch. f. otol. New York, Dez. 1908. Ein Fall von Thrombophleb. des Sin. cavern. b. Empyem d. Kreil-u. Siebbeinhöhle.

Moschew, M. P. (Boston). D. Orbitalweg .. Sinus cavern. (The Laryng. St. Louis, Aug. 1914).

Krebs, Trochlearlähmung b. Kieferhöleneiterung (Therap. Mon. Schr. Nr. 9).

Kander, (Karlsruhe). Üb. d. intercraniellen Erkrankgungen b. Enpyem d. Ke:Ibeinhöıle (bruns Beitr. z. klin. Chir. Bd. 36. H. 1.)

Caradt, (Delmiro), Empyem d. Sin front. m. diff. Phlegm. u. Gangr. d. ob. Augenlides m. Keratoiritis u. Erysipel kompl, (Revist. d. Med, chirurg. Barcelona, April 1903.)

Lafarre, (Brïssel), Ber. über d. Jahresvers. d. belg. oto-rhino-laryng. Gesellsch. (Juni 1902 litüssel). Orbit. Komplik. im Anschluss an einer Sin. front. mit Caries des Orbitaldaches. 
Harald, Wilson, (Detroit), Sinusthromb. Kleinhirnabsc. u. Meningitis im Anschl. an eine Osteomyelitis d. Keilbeins. (Arch. f. Otolog., Vol. 31. Nr. 6. Dez. 1902).

Uchermanu, Cavern. Thrombose aus Sin. spenoid, (Sitz d, oto-laryng. Vereins, Gesellsch. Berichte 1920, Febr. 26).

Ga1tung, Über einen Fall. v. Thrombose im Sin. cavern aus Nase (Sin. sphenoid). Stzg. d. oto-laryng, Vereins 1920, Febr.

Rudger, T, Ritchie, Cavern. Thrombose (The Journ. of Laryng. and otol. Apr. 1921.

Hey-Abero, Thrombose of the cavernosus (Acta otolaryng., Stockholm, 1921, Bd. III. h. 1-2.) aus: doppelseit. Keilbeinhöhleneiterung. 